\title{
Hypotensive infarction of the spinal cord
}

\author{
U Singh MB BS DPMR Diplomate NB (PMR) ${ }^{1} \mathrm{~J}$ R Silver MB BS FRCP(Ed) \\ FRCP(Lond), ${ }^{2}$ The late N C Welply MBE MRCS LRCP DA ${ }^{3}$
}

\begin{abstract}
${ }^{1}$ Associate Professor, Department of Rehabilitation All India Institute of Medical Sciences, New Delhi-110029, India; ${ }^{2}$ Emeritus Consultant in Spinal Injuries, ${ }^{3}$ Retired Consultant Anaesthetist, National Spinal Injuries Centre, Stoke Mandeville Hospital, Aylesbury, Bucks HP21 8AL, England.
\end{abstract}

Four patients developed infarction of the lumbar cord as a result of induced hypotension. All patients showed a lesion in the territory of the artery of Adamkiewicz. The pathophysiology of the blood supply of the spinal cord is discussed, and in particular the anatomy of the artery of Adamkiewicz. The reason why this artery is so vulnerable to hypotension is discussed.

Keywords: spinal cord infarction; hypotension; artery of Adamkiewicz.

\section{Introduction}

The blood supply of the spinal cord was first described in 1882 by Adamkiewicz. ${ }^{1}$ Further studies were carried out by Bolton (1939), ${ }^{2}$ Cook (1959), ${ }^{3}$ Garland (1966) ${ }^{4}$ and Henson (1967). ${ }^{5}$ It has been recognised that the anterior spinal artery territory is vulnerable if one of the main contributary vessels is damaged, and the complication of paraplegia as the result of operations upon the arch of the aorta and upon the aorta have been described. The development of paraplegia as the result of a reduction in the perfusion pressure was less well recognised, so that in 1974,11 patients, who were seen between 1965 and 1972 and who developed paraplegia, were described. ${ }^{6}$ In only one of the patients was the paraplegia due to blockage of the aorta by a saddle embolus; in the majority of the other patients the paraplegia resulted from a fall in the perfusion pressure; nine of the 11 patients were extremely ill due to coronary thrombosis or severe blood loss. Four of these died within a short time, either during the initial admission or subsequently. Only two of the younger patients, whose low blood pressure was the result of suicide attempts, had normal cardiovascular systems prior to the infarction of the cord. Thus the clinical picture of the spinal cord infarction was modified by the precipitating medical condition.
A further four patients are now described who were completely fit prior to the development of spinal cord infarction, in whom the fall in blood pressure was caused solely by the drop in the perfusion pressure. This condition was not modified by an accompanying severe medical condition or severe blood loss and further delineates the role of hypotension, its severity, its duration and its relationship to the loss of circulating blood volume to the spinal cord infarction.

\section{Case histories}

\section{Case 1}

A 59 year old woman $(57.1 \mathrm{~kg})$ was admitted for cholecystectomy. She had a previous history of four uneventful operations carried out under general anaesthesia (GA), but was otherwise fit apart from having a blood pressure (BP) of $180 / 90 \mathrm{~mm} / \mathrm{Hg}$ and a slightly abnormal electrocardiograph (ECG). Premedication of papaveretum (Omnopon) $20 \mathrm{mg}$ and atropine $0.6 \mathrm{mg}$ was given intramuscularly (IM) 2 hours prior to surgery. GA was induced with thiopentone $250 \mathrm{mg}$ and suxamethonium $75 \mathrm{mg}$ followed by nitrous oxide and halothane. In addition an epidural cannula was placed at the level of T6-7 interspace to inject $10 \mathrm{ml} 0.5 \%$ bupivacaine without adrenaline. During anaesthesia there was a fall in BP from $130 / 80 \mathrm{~mm} / \mathrm{Hg}$. at the beginning of the anaesthetic, to $80 / 50 \mathrm{~mm} / \mathrm{Hg}$, which was treated with $500 \mathrm{ml}$ of dextran and one litre of Hartmann's solution. Peroperative cholangiography was done and the 
removal of the gall bladder was uneventful. The $\mathrm{BP}$ was normal at the end of the anaesthetic.

She regained consciousness, was drowsy, but was orientated in time and space and obeyed commands. Her cardiovascular system was satisfactory. During the postoperative period she was given top-up doses of $6 \mathrm{ml} 0.5 \%$ bupivacaine repeated 3 and 17 hours after surgery, with instructions for the $\mathrm{BP}$ to be recorded every 10 minutes for half an hour after each dose. About 8 hours after the operation she felt nauseated and was given perphenazine $5 \mathrm{mg}$ IM, and with further vomiting this dose was repeated 7 hours later. About 18 hours postoperation, ie an hour after the last top-up of bupivacaine, she was made to sit up in bed by a nurse for a blanket bath, and became pale with a weak pulse and an unrecordable BP. About 5 minutes after resuscitation was commenced, she had a BP of $60 \mathrm{~mm} / \mathrm{Hg}$ systolic, and after 20 minutes the BP was $100 / 60 \mathrm{~mm} / \mathrm{Hg}$. She was deeply unconscious, flaccid in all four limbs and areflexic for about 2 hours when she started responding to painful stimuli. Some movements returned to her upper limbs after 8 hours. She recovered full consciousness in about 24 hours and was found to have an impaired memory, reduced visual acuity, and partial paralysis of all four limbs. The motor loss was incomplete at C6 level, there was a sensory level at T1, and there was no control over her bladder and bowel. Haematological and biochemical investigations were normal. $\mathrm{X}$-rays of the spine showed osteoarthritic changes in the lower thoracic spine but without any definite abnormality of the spinal canal.

She was admitted to the National Spinal Injuries Centre at Stoke Mandeville Hospital (NSIC) about $2 \frac{1}{2}$ months later for rehabilitation, and made a considerable recovery of motor power and sensation in the upper limbs. At the time of discharge about 10 months later, she was a cheerful and cooperative lady, not fully oriented to time and space, with impaired memory of recent and past events, impaired mathematical functions and reduced visual acuity. The upper limbs were normal in motor power with mild ataxia. In the lower limbs there was a flaccid paralysis with a flicker of voluntary movement in the right big toe only. Pain sensation was diminished below T10; touch and posterior column sensation were preserved all over. The reflexes were not elicitable in the lower limbs. Bladder and bowel sensation and control were absent, and she had an indwelling catheter in place. She was independent in propelling a manual wheelchair but needed help for most activities of daily living
(ADL). Subsequently, over the ensuing years her upper limbs became normal but she has remained with a dense paralysis of her lower limbs. Posterior column sensation was preserved. In 1993 she is living independently at home.

\section{Case 2}

A 71 year old man $(82.55 \mathrm{~kg})$ was admitted with a diagnosis of arthritis of the left hip for a Charnley hip replacement, with a history of prior uneventful surgery for a left inguinal hernia. There was evidence of arteriosclerosis on examination of fundi. There was a history suggestive of spinal cord claudication in the form of a feeling of stiffness and clumsiness of the legs after climbing several floors. The BP on admission was $150 / 100 \mathrm{~mm} / \mathrm{Hg}$. He was given premedication of nembutal $100 \mathrm{mg}$ and promethazine hydrochloride (Phenergan) $25 \mathrm{mg}$ orally 2 hours before operation. $\mathrm{He}$ was induced with methohexitone $100 \mathrm{mg}$ and suxamethonium $50 \mathrm{mg}$ IV and was ventilated. A lumbar epidural block at L4-5 interspace was performed with $20 \mathrm{ml} 1.5 \%$ lignocaine and $20 \mathrm{ml} 0.5 \%$ bupivacaine. He was given $900 \mathrm{ml}$ dextrose saline during the operation. Pethidine $50 \mathrm{mg}$ IV was given just before leaving the theatre. There is no record of the BP or of blood loss during the operation. On return to the ward his $\mathrm{BP}$ was $90 / 60 \mathrm{~mm} / \mathrm{Hg}$ which rose to $135 / 85 \mathrm{~mm} / \mathrm{Hg}$ about 6 hours after the operation. After the operation he was found to be confused and unable to move both lower limbs. On examination he had a total loss of joint sensation below $T_{12}$, while other modalities of sensation were preserved. $\mathrm{He}$ was areflexic in his lower limbs with no control or sensation of bladder and bowel. He started to show recovery of joint sensation in about 24 hours. $\mathrm{X}$-rays of the spine and myelography were normal, as were haematological and biochemical investigations. He showed some further improvement with the passage of time.

$\mathrm{He}$ was admitted to the NSIC about 2 months after the onset of the paraplegia to commence a rehabilitation programme, and was discharged about $4 \frac{1}{2}$ months later. Bladder and bowel sensation returned but without control and he was put on condom drainage. $\mathrm{He}$ was wheelchair independent for most ADL and could ambulate a little. There has been a gradual return to power in the gracilis, sartorius and quadriceps of the left lower limb, and sensory appreciation has improved. He has returned to the NSIC for various procedures and 
in 1993 he was alive but was suffering from an intercurrent medical condition resulting in anaemia which has required hospital admission.

\section{Case 3}

A 49 year old woman $(55.5 \mathrm{~kg})$ was admitted to a private nursing home for cosmetic surgery for a face lift. The BP recorded on two occasions before surgery was $124 / 80 \mathrm{~mm} / \mathrm{Hg}$ and $170 / 100 \mathrm{mg} / \mathrm{Hg}$. She was otherwise a healthy lady with a previous history of a hysterectomy, which was uneventful. She was given local analgesia for the cosmetic surgery with $80 \mathrm{ml}$ $0.5 \%$ bupivacaine and $16 \mathrm{ml} 2 \%$ lignocaine subcutaneously along with $20 \mathrm{mg}$ of Valium IV. After the injection of local anaesthetic she developed fits and muscle twitching which lasted for about half an hour; she maintained a satisfactory pulse. Suddenly she became unconscious and had a cardiorespiratory arrest. External cardiac massage was commenced and oxygen was given via a mask. An anaesthetist was called. The monitor, when attached, showed very slow ventricular rhythm. She was intubated, ventilated and given $200 \mathrm{mg}$ sodium bicarbonate, $10 \mathrm{ml}$ calcium gluconate and $1 \mathrm{ml}$ 1:1000 adrenaline IV, which led to a good pulse and a sinus rhythm along with spontaneous breathing. A few minutes after resuscitation the $\mathrm{BP}$ returned to $90 \mathrm{~mm} / \mathrm{Hg}$ systolic, she was grade IV unconscious, had no spontaneous movements, the pupils were small, equal and reacting to light. An hour later she started responding to commands but was kept sedated and on a ventilator. She was later transferred to NSIC with a stable cardiorespiratory function. The cranial nerves were normal, lower limbs were flaccid with no motor power, sensory appreciation was impaired to the modalities of touch, pain and temperature below T6. Posterior column function was preserved and the reflexes were not elicitable in the lower limbs. There was no sensation or control of bladder or bowel function and she was catheterised. X-rays of the spine showed minor 'changes' at the level of T7-8 which were not thought to be recent. She was fully conscious and was taken off the ventilator the following day, but did not show much further recovery in her neurological status. At the time of her discharge about 6 months after the onset of the paraplegia she had no motor power below $\mathrm{T} 7$ and was areflexic in the lower limbs. The modalities of pain, touch and temperature appreciation were lost below T6 and posterior column sensation was preserved. She had full sensation and con- trol of her bladder apart from stress incontinence. Bowel sensation was present but there was no control. She was independent in a wheelchair for almost all ADL. She was orientated in time and space, and in 1993 she is still alive and well and living on her own.

\section{Case 4}

A 51 year old married woman $(56 \mathrm{~kg})$ was admitted for cosmetic surgery for a face lift operation. She had no previous illnesses apart from a minor back strain. She did not smoke or drink. Preoperatively her blood pressure was $140 / 90 \mathrm{~mm} / \mathrm{Hg}$. The operation was carried out under a general anaesthetic. The blood pressure was reduced by a combination of the following drugs: Pentolinium, (a ganglion blocker), tubocuravine (a muscle blocker), Inderal (propanolol, a beta blocker), halothane and suxamethonium. In addition she was tilted to $70^{\circ}$ to further lower the blood pressure. During the anaesthetic her blood pressure fell to 47 systolic, 25 diastolic $\mathrm{mm} / \mathrm{Hg}$ and remained at that level for $2 \frac{1}{2}$ hours after the commencement of the operation. During the operation her legs became cold and the anaesthetist gave her $8 \mathrm{mg}$ dexamethasone IM but the operation continued. Four hours after the operation commenced she recovered consciousness and complained of numbness in her lower limbs and some pain in her heels. Her blood pressure was $90 / 50 \mathrm{~mm} / \mathrm{Hg} ; 11$ hours after the operation it was $100 / 60 \mathrm{mmHg}$ and by the next morning it had risen to $140 / 90 \mathrm{~mm} / \mathrm{Hg}$, her preoperative level. At that stage she observed that she could not pass urine and could not move her lower limbs or get out of bed. That evening she was examined by a consultant neurologist who found that there was no abnormality in her cerebral state, but that the tendon reflexes in her upper limbs were brisk. She had diffuse motor weakness of her lower limbs, but preservation of the knee jerks. The ankle jerks were absent. The right plantar reflex was equivocal, the left was extensor. Sensation was impaired over her buttocks. Blood results were normal and $\mathrm{x}$-rays of the spine showed degenerative changes. Subsequently she was investigated by means of an MRI scan which showed swelling of the lumbar cord with segmental extension up to the thoracic cord suggestive of ischaemia. There was virtually no recovery of power and she was discharged home some 3 weeks later to attend as an outpatient for an active programme of rehabilitation. Further investigations were carried out in the form of a myelogram, 
which showed no abnormality, and nerve conduction studies, which were normal. Some 5 years after the episode she is still severely disabled with little change in her neurological condition and little neurological recovery. She walks with a high stepping gait, and is incontinent of bladder and bowel with almost no sexual function. Cranial nerves are normal, upper limbs are normal but in the lower limbs there is weakness of the psoas, the hamstrings and the long flexors of the toes. There is no motor power below this level. There is widespread fasiculation of all of the muscles in her lower limbs. The knee jerks are preserved, the ankle jerks are absent, plantar reflexes are absent, and the anal reflex is absent. Sensation is impaired below L2.

\section{Discussion}

The abrupt onset of a clearly defined incomplete lesion of the lumbar spinal cord in four previously fit adults (apart from one patient who had a previous history suggestive of cord claudication) with the accompaniment of transient cerebral anoxic damage following an anaesthetic during which there was significant and prolonged hypotension is of an identical pattern to 11 patients previously described $^{6}$ and establishes that it was related to the operation and was due to infarction of the spinal cord.

The pattern of recovery was variable. No patient recovered completely. One of the patients showed a significant and gradual recovery of motor power, in the others motor deficit remained constant. Recovery of sensory function was more marked, especially posterior column function, which was either preserved or recovered completely. The sensation of the bladder and bowel was initially lost in all four patients, but two had shown substantial recovery at the time of their last examination at the beginning of 1993 and, apart from one elderly patient with an intercurrent medical condition, all the patients were living at home and the level of independence in all four patients was satisfactory.

The patients all showed the features of a lesion in the territory of the artery of Adamkiewicz. These patients came under the care of the physicians at the National
Spinal Injuries Centre at a variable time after the operation. They had been seen by numerous neurologists at the receiving hospitals and had been comprehensively investigated. No further investigations were thought to be necessary, or were available. Angiography of the spinal cord does not always demonstrate the spinal vasculature and in the presence of a thrombotic lesion would in any event be contraindicated. In one patient, the one seen most recently, an MRI study was carried out. This showed a swollen cord in the lumbar region. This investigation would have been valuable had it been available for the other patients as it is noninvasive, and were such patients to be seen today, this investigation would be carried out. In order to delineate the pathogenesis of this condition it is necessary to understand the blood supply of the spinal cord.

The major blood vessels that supply the cord are derived in the cervical region from the subclavian and vertebral arteries. The thoracic cord is supplied by small segmental vessels arising directly from the aorta, and the lumbosacral cord derives its blood supply from the internal iliac arteries.

The arteries of the cord themselves are three major longitudinal vessels derived from the vertebral artery within the skull: two small posterior spinal arteries up to $0.8 \mathrm{~mm}$ in diameter and a large single median anterior vessel up to $1.2 \mathrm{~mm}$ in diameter. Each gives rise to branches which make communication between the longitudinal vessels. The anterior spinal artery gives rise to perforating vessels within the spinal cord. Each of the main longitudinal vessels is reinforced with blood by a number of feeding vessels arising from the segmental arteries. The posterior spinal segmental vessels are smaller than the anterior ones.

The anterior spinal artery receives three or four major contributory vessels. Their distribution is variable. There is usually one in the cervical, two in the thoracic and one in the lumbar region. The diameter of these vessels is comparable with that of the anterior spinal artery.

Romanes ${ }^{7}$ made a particular study of the large lumbar vessel of Adamkiewicz. He studied 22 spinal cords, and in 19 of these 
this vessel arose on the left hand side and in the remaining three cases it was on the right hand side. In the majority of cases (11) it arose between $\mathrm{T} 10$ and $\mathrm{T} 11$ and in the remaining eight cases it arose between $\mathrm{T} 6$ and L3. He therefore concluded that there was always a large vessel in the lumbar region but its exact place of origin was variable.

There are thus two circulations: an anterior circulation derived from the anterior spinal artery reinforced by four large feeding vessels which supply the anterior two thirds of the spinal cord, and a smaller posterior circulation which supplies the posterior third of the spinal cord.

There is a rich anastomosis in only one region, that is around the tip of the cauda equina. This is comparable to the anastomosis of the circle of Willis, but otherwise there is no anastomosis either circumferentially or within the spinal cord.

This has been verified by injection techniques, and selective injections into the single radicular artery show that in most cases the entire spinal cord cannot be filled by this procedure. ${ }^{8}$ The pattern of the arterial supply to the spinal cord is via a system of longitudinal anastomoses reinforced at various segmental levels. This protects the neural tissue against ischaemia. The dominant role of the longitudinal circulation is crucial. There is controversy as to whether the long anastomosis is adequate at the watershed (the midthoracic region) and whether ischaemic changes are found at this point. The intramedullary communications may only be important for slower circulatory adjustments, since their calibers are inadequate for a sudden intake of large volumes of blood.

Thron $^{8}$ has stressed that the segmental arterial supply may function well in situations demanding rapid compensation but it cannot function in haemodynamic crises. $\mathrm{He}$ believed that this explained the verified necroses of the anterior horn cell of the spinal cord following circulatory arrest, which he considered to be consequently the most vulnerable structure of the spinal cord.

Compared with cerebral strokes, spinal strokes are remarkably rare. The first description was by Spiller in $1909 .{ }^{9} \mathrm{He}$ des- cribed a patient with a postmortem study in which a thrombosis of the anterior spinal artery was demonstrated. He discussed two further cases.

Bastian in $1910^{10}$ referred to previous cases and presented further cases. Schaller et al (1932) ${ }^{11}$ described two further cases without postmortems. Zeitlin \& Lichtenstein $(1936)^{12}$ described a further case with a postmortem. In that case there was severe atheroma of the branches of the anterior spinal artery with a softening of the anterior part of the spinal cord. This was of spontaneous onset. There were further accounts by Silver \& Buxton $^{6}$ with two postmortems (1974) where there was no blockage demonstrable of the anterior spinal artery but the cord showed infarction. Hughes $(1977)^{13}$ described two patients with postmortems in which there was infarction of the cord but again the spinal artery was not blocked. Blumbergs \& Byrne (1980) ${ }^{14}$ described a case of infarction of the cord without there being blockage of the spinal arteries. Satran ${ }^{15}$ published a review article in 1987. He pointed out that cardiac arrest could be the cause of infarction of the cord without there being a blockage of the anterior spinal artery.

In all of these accounts the patients presented the features of anterior spinal artery thrombosis. The cord was infarcted in the distribution of the anterior spinal artery. These descriptions are characterised by their rarity and the fact that few of them actually observed thrombosis of the anterior spinal artery. None drew attention to the role of the artery of Adamkiewicz.

One of the authors saw a patient who had a lesion of the artery of Adamkiewicz due to a direct puncture of the aorta for an aortogram. This compromised the circulation of the cord and produced an asymmetrical loss of power and sensation in the lower limbs with impairment of sensation as high as T5 which subsequently regressed with preservation of bladder function. This reflected the damage to the anterior part of the cord in the territory supplied by the artery of Adamkiewicz. We prefer to use the term lesion of the artery of Adamkiewicz, rather than the broad descriptive term anterior spinal artery thrombosis, which in 
any case lacks anatomical or diagnostic verification.

The four patients presented here showed these features. There was preservation of bladder function with sacral sparing which can be explained by the rich anastomoses around the tip of the conus. The involvement of the anterior two thirds of the cord is due to the fact that the blood supply of the anterior two thirds is derived from the anterior spinal artery and emphasises the contribution that the artery of Adamkiewicz makes. The gradual recovery over 2 years of some motor power and some sensory appreciation was found at the periphery where the posterior circulation is taking over. The upper limit of impairment of sensation was at T5 indicating that there was a partial watershed at this level.

The common aetiological feature in all of these patients was that all had a degree of hypotension. The hypotension was induced as the result of therapy, in contrast to the previous series where the hypotension was the result of illness or massive blood loss. It is recognised practice to give either a spinal or epidural anaesthetic to reduce painful stimuli and thus reduce the blood loss rendering a patient unconscious only from a sleep dose of anaesthesia. This can reduce the amount of general anaesthetic given.

When a spinal anaesthetic is administered for the relief of pain not only does it eliminate painful afferent stimuli but it also paralyses the sympathetic. The circulation may be perfectly adequate in the recumbent position, but if the patient is suddenly sat up (case 1), or turned, as in a further case not in this series, where the patient had been given a spinal anaesthetic to relieve pain, then postural hypotension will result. The reason for this is that only a proportion of the vascular control resides within the sympathetic, the rest is intrinsic to the musculature of the blood vessels and the circulation may be perfectly adequate to perfuse the vital organs and brain and spinal cord in the recumbent position but not when the patient is sat up.

We attempted to determine the relationship between infarction of the cord from hypotension which was caused by paralysis of the sympathetic, or acute heart failure, and to separate it from hypotension and anoxia due to severe blood loss. This problem was previously encountered when studying patients with infarction of the spinal cord due to traumatic rupture of the aorta. In this series ${ }^{16}$ the records did not always allow us to determine accurately the amount of blood loss, but in at least three of the patients the infarction of the cord was due to hypotension alone and not due to severe blood loss.

The most interesting question to be answered is "Why is the spinal cord and in particular the territory supplied by the artery of Adamkiewicz so vulnerable to hypotension?' Also, 'Which is more vulnerable, the brain or the spinal cord?' The artery of Adamkiewicz is vulnerable because it is the last major vessel feeding the spinal cord and it makes an acute angle of entry which may contribute to its vulnerability. There are no functioning anastomoses between the anterior and posterior circulations even though communications may be demonstrated. The artery of Adamkiewicz is effectively an end artery. It is striking that in the reports quoted there has been no blockage of the anterior spinal artery but the cord has been infarcted by a drop in the blood pressure.

Blumbergs \& Byrne ${ }^{14}$ addressed this problem in their case, which was due to clamping of the aorta for an operation for coarctation of the aorta. They found that the caudal cord is critically dependent upon the longitudinal vascular plexus for its blood supply and because of the ostial narrowing of the lumbar and iliolumbar vessels when hypotension occurs, the sacral cord being the most distal showed the most severe damage. They found no involvement of the T4 level which was believed to be the site of greatest vulnerability in hypoperfusion syndromes. The central grey matter was necrotic, which they believed demonstrated the selective vulnerability of this tissue within the spinal cord.

Kobrine et $a l^{17}$ have addressed this problem experimentally to determine the relative vulnerability of the brain and spinal cord to ischaemia. They carried out studies of the blood flow in the brain and spinal cord in five healthy monkeys. They found 
that the lower limits of autoregulation of the blood flow to the brain were somewhat more stable than in the spinal cord and more refractory to the effects of hypotension. They found that in the animals, the spinal cord blood flow and the cerebral blood flow began to fall when the mean arterial blood pressure was lowered to $50 \mathrm{~mm}$ mercury, and that at $25 \mathrm{~mm}$ mercury the mean arterial blood flow was immeasurable in all animals. When it is considered that in these patients blood pressures of this order were recorded it is not surprising that the spinal cord demonstrated infarction.

However, in healthy monkeys with normal cerebral arteries the degree of hypotension needed to achieve critical hypoperfusion to such a degree that consciousness would be lost or severely depressed should be contrasted to subjects where the vessels can be occluded by arteriosclerosis or where autoregulation is impaired. The threshold in vulnerable areas might be reached with more modest, sustained, or even intermittent falls in blood pressure. Thus it would seem that autoregulation persists and is more effective in the brain than in the spinal cord and that under certain circumstances the spinal cord would be more vulnerable to a fall in the blood pressure. Furthermore, it would seem that the absence of blockage of the vessels in these postmortem series would point to the fact that this was ischaemia and not actual occlusion of the vessels that has caused damage to the spinal cord.

It is striking that since the last paper $^{6}$ reporting 11 cases due to reduction in blood pressure there have been few reports of a similar nature and the incidence of infarction of the cord and its relationship to cerebral infarction is an interesting one. Cerebral strokes are common and spinal strokes are not. It is difficult to visualise the spinal vessels, they are not readily seen at postmortem due to technical difficulties. Ideally the spinal vessels should be dissected back to the aorta but this can only be carried out at specialised centres and would only reveal infarction due to vascular insufficiency. Angiography still cannot visualise the vessel of Adamkiewicz and in any event would be contraindicated. MRI scans, which are now freely available, should provide further information on the subject. It may well be that the occurrence of postoperative paraplegia that occurs in neurosurgical and other orthopaedic centres due to surgery upon the spinal cord, the intervertebral discs and scoliosis are not due to direct damage to the blood vessels of the cord but are due to prolonged recumbency, and episodes of hypotension which cause infarction of the cord.

Thus hypotension should be avoided and the blood pressure regularly monitored. The blood pressure should be maintained throughout surgical procedures since infarction of the cord can occur in healthy subjects with minor hypotensive episodes and when the subject is elderly or some vascular impairment is there already, then even greater vigilance must be exercised.

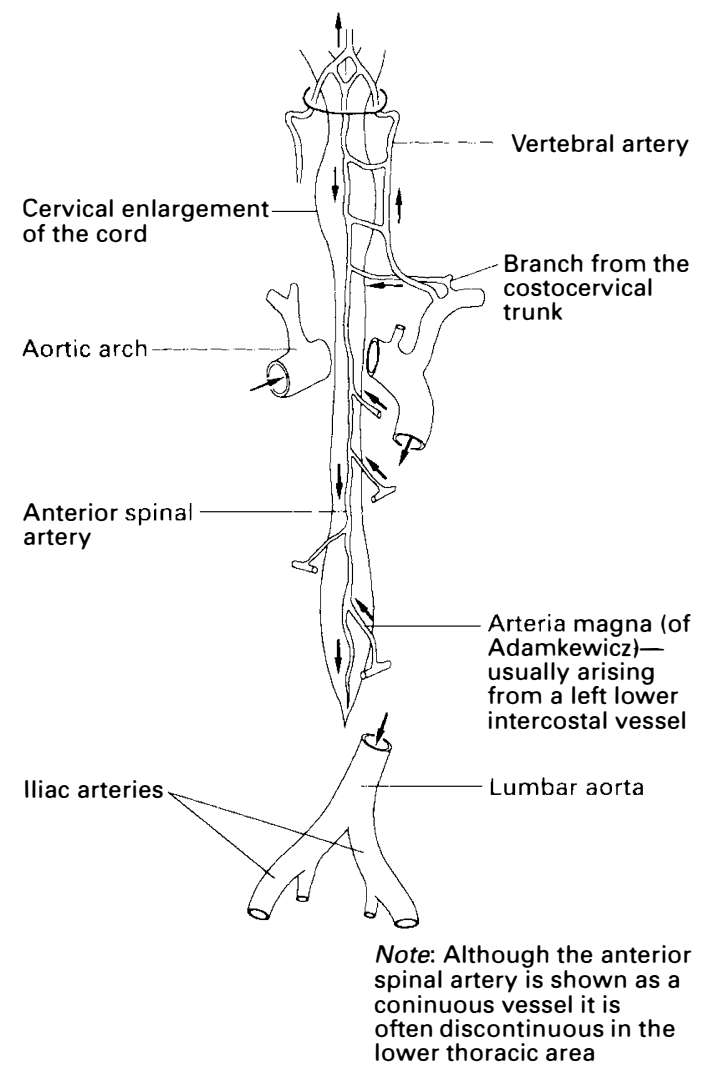

Figure 1 Blood supply to the spinal cord. Note the arteria magna of Adamkiewicz. 


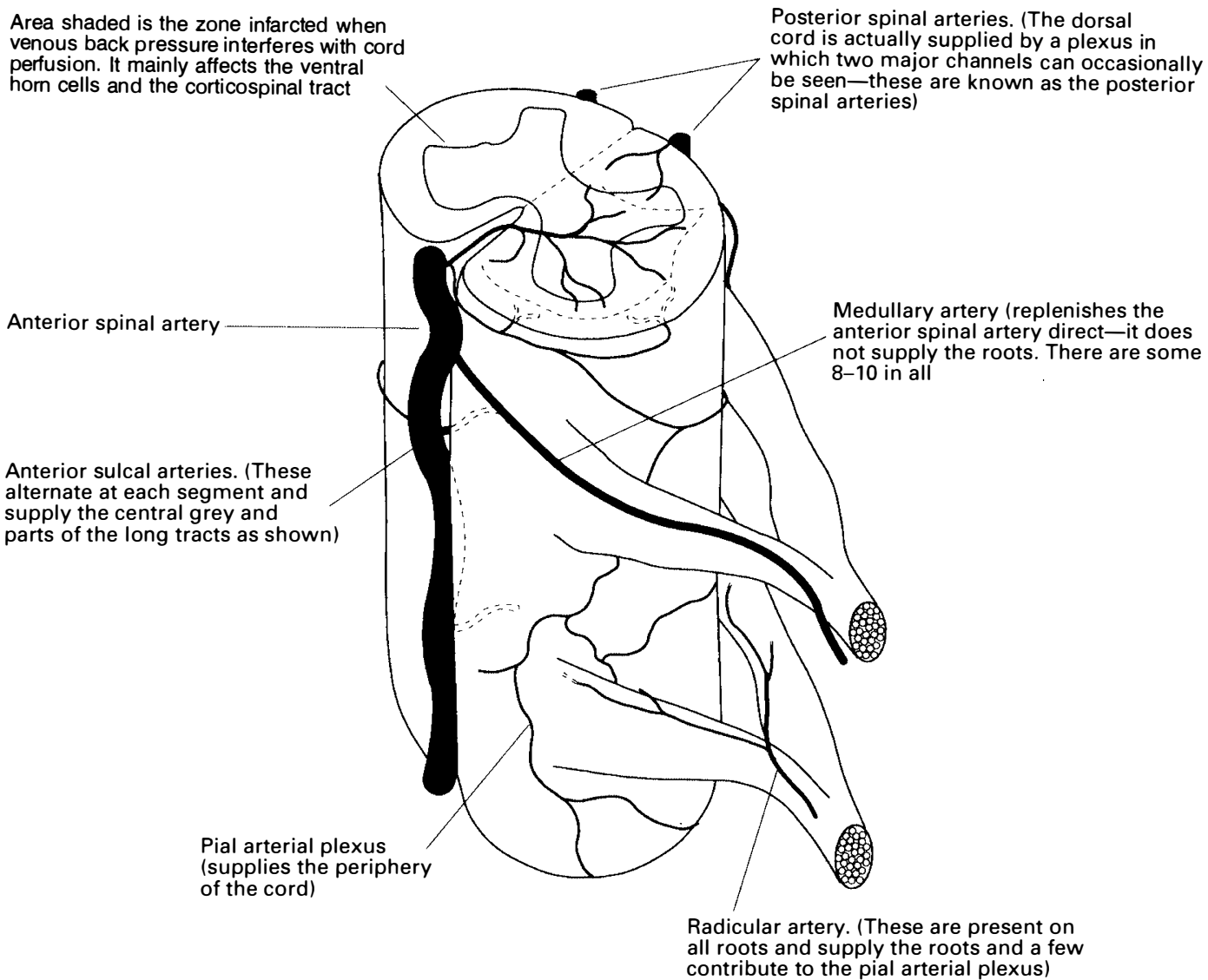

Figure 2 Segmental blood supply of the spinal cord supplying the lumbar area. The damage is due to infarction. The spinal arteries may give rise to sparing of the posterior column.

\section{Acknowledgements}

We thank the Association of Commonwealth Universities, 36 Gordon Square, London, for sponsoring Dr U Singh, who worked as a Commonwealth Medical Fellow at the National Spinal Injury Centre, Stoke Mandeville Hospital, Aylesbury. Acknowledgements are also

due to Dr John Patten and Springer Verlag London Ltd for the use of Figures 1 and 2 from Neurological Differential Diagnosis (1977). We are grateful to $\mathrm{Dr} \mathrm{H}$ L Frankel, $\mathrm{Mr} \mathrm{B} \mathrm{P}$ Gardner and Mr I Nuseibeh for permission to study their patients.

\section{References}

1 Adamkiewicz A (1882) Die Blutegefaasse des menschlichen Ruckenmarkes: II. Die Gefasse der RuckenmarksoberFlache. Sber Akad Wiss Wien, Abt II 85: 101-130.

2 Bolton B (1939) The blood supply of the human spinal cord. J Neurol Neurosurg Psychiatry 2: 137-148.

3 Cook AW (1959) Occlusion of the abdominal aorta and dysfunction of the spinal cord; a clinical syndrome. Bull NY Acad Med 35: 479-489.

4 Garland HJ, Greenberg J, Harriman DEF (1966) Infarction of the spinal cord. Brain 89: 645-662.

5 Henson RA, Parsons M (1967) Ischaemic lesions of the spinal cord: an illustrated review. $Q$ J Med 35: 205-222.

6 Silver JR, Buxton PH (1974) Spinal Stroke. Brain 97: 539-550.

7 Romanes GJ (1964) The arterial blood supply of the human spinal cord. Paraplegia 2: 119-206. 
8 Thron AK (1988) Vascular Anatomy of the Spinal Cord, Neuroradiological Investigations and Clinical Syndromes. Springer-Verlag, Wien, New York: 38.

9 Spiller WG (1909) Thrombosis of the cervical anterior median spinal artery; syphilitic acute anterior poliomyelitis. J Nervous Mental Dis 36: 601-613.

10 Bastian HC (1910) Observations on thrombotic softening of the spinal cord as a cause of so-called 'acute myelitis'. The Lancet, November 26, 1531-1534.

11 Schaller WF, Roberts AM, Stadtherr EF (1932) Acute myelitis (myelomalacia) syndrome of occlusion of the anterior spinal artery at the fifth cervical cord segment. JAMA 99: 1572-1575.

12 Zeitlin H, Lichtenstein BW (1936) Occlusion of the anterior spinal artery: clinicopathologic report of a case and a review of the literature. Arch Neurol Psychiatry 36: 96-111.

13 Hughes JT (1977) Spinal cord involvement by C4-C5 vertebral subluxation in rheumatoid arthritis: a description of two cases examined at necropsy. Ann Neurol 1: 575-582.

14 Blumbergs PC, Byrne E (1980) Hypotensive central infarction of the spinal cord. J Neurol Neurosurg Psychiatry 43: 751-53.

15 Satran R (1987) Spinal cord infarction. Curr Concepts Cerebrovasc Dis-Stroke 22: 13-17.

16 Jamous MA, Silver JR, Baker JHE (1992) Paraplegia and traumatic rupture of the aorta: a disease process or surgical complication? Injury 23: 475-478.

17 Kobrine AI, Evans DE, Rizzole HV (1980) Relative vulnerability of the brain and spinal cord to ischemia. J Neurol Sci 45: 65-72. 